\title{
Visceral disseminated varicella zoster infection: a rare cause of acute abdomen in a patient with well-controlled diabetes mellitus - a case report
}

\author{
Daisuke Mizu* ${ }^{*}$, Haruka Nishida, Yoshinori Matsuoka and Koichi Ariyoshi
}

\begin{abstract}
Background: Visceral disseminated varicella zoster virus (VZV) infections frequently affect immunocompromised patients. Diabetes mellitus has been associated with VZV infection, and most cases of disseminated infection involve patients with poorly controlled blood glucose levels. It initially presents as severe abdominal pain, which is evaluated as an acute abdomen, however, the cause is typically unidentified due to unremarkable computed tomography (CT) findings. We report a case of visceral disseminated VZV infection in a patient with well-controlled diabetes mellitus with fat stranding around the celiac and superior mesenteric artery on CT.
\end{abstract}

Case presentation: A 61-year-old Japanese woman with well-controlled diabetes mellitus presented to the emergency department with severe abdominal pain that gradually worsened. She had stable vital signs and skin rashes, suggestive of varicella. Abdominal CT showed fat stranding around the celiac and superior mesenteric arteries. The patient tested positive for the VZV antigen and was diagnosed with a visceral disseminated VZV infection. Acyclovir was administered, and the patient was discharged on the 14th day.

Conclusions: Visceral disseminated VZV infection may affect patients with well-controlled diabetes mellitus and causes acute abdomen. Periarterial fat stranding on $C T$ is associated with abdominal pain due to visceral disseminated VZV infection.

Keywords: Acute abdomen, Case report, Computed tomography, Diabetes mellitus, Disseminated varicella zoster infection, Periarterial fat stranding, Varicella zoster virus

\section{Background}

Varicella zoster virus (VZV) infections have a severe clinical course in immunocompromised patients. Specifically, patients who underwent bone marrow transplantation have the highest risk of developing disseminated infection [1]. Abdominal pain is often the initial symptom of visceral disseminated VZV infection, and the skin

\footnotetext{
*Correspondence: water@kcho.jp
}

Department of Emergency Medicine, Kobe City Medical Center

General Hospital, 2-1-1, Minatojimaminami-machi, Chuo-ku, Kobe-shi,

Hyogo 650-0047, Japan rash, characteristic of VZV infection, appears after than abdominal pain $[2,3]$. Therefore, this disease is difficult to diagnosis early. However, delayed treatment can lead to multiorgan failure [4], and misdiagnosis in the emergency department negatively affects patient prognosis. In immunocompromised patients, visceral disseminated VZV infection should be considered a differential diagnosis for acute abdomen due to the high risk of developing the disease. Diabetes mellitus (DM) is also associated with VZV infection, and most cases of disseminated VZV infection involve patients with poorly controlled blood 
glucose levels [5, 6]. Visceral disseminated VZV infection rarely affects patients with well-controlled DM.

The cause of severe abdominal pain, associated with visceral disseminated VZV infection, remains unclear with negative imaging findings $[7,8]$. Recently, periarterial fat stranding around the celiac artery (CA) and superior mesenteric artery (SMA) was reportedly confirmed by computed tomography $(\mathrm{CT})[8-10]$.

We report a case of visceral disseminated VZV infection in a patient with well-controlled DM with severe abdominal pain and periarterial fat stranding on $\mathrm{CT}$.

\section{Case presentation}

A 61-year-old Japanese woman with postoperative ovarian cancer and DM, controlled with oral antidiabetic medications or drugs, presented to the emergency department (ED) with a complaint of abdominal pain that worsened over four days. She underwent surgery for ovarian cancer 11 years ago and received no chemotherapy as the disease was still at an early stage. She had been followed up as an outpatient for approximately six years and was confirmed to have no recurrence. She had other medical histories of hypertension and hyperlipidemia, with no recent history of taking any steroids or immunosuppressive medications.

On the day of her visit to the ED, skin rashes were noted on her face, abdomen, and thighs. Within the four days between the onset of abdominal pain and the patient's visit to the ED, she had experienced few episodes of vomiting. Abdominal examination revealed tenderness in the epigastric area without muscular guarding or rebound tenderness. The skin rashes were accompanied by blisters. The patient had a Glasgow Coma Scale of 15 (E4V5M6), blood pressure of 139/81 $\mathrm{mmHg}$, heart rate of $76 / \mathrm{min}$, respiratory rate of $16 / \mathrm{min}$, body temperature of $35.9{ }^{\circ} \mathrm{C}$, and oxygen saturation level of $100 \%$ (room air). Blood tests in the ED showed a slight increase in the C-reactive protein level, as shown in Table 1. Abdominal contrast-enhanced $\mathrm{CT}$, which was performed to evaluate the abdominal pain, showed periarterial fat stranding around the CA and SMA (Fig. 1a, b). No other abnormal findings were observed, and the patient tested positive for the VZV antigen and was diagnosed with visceral disseminated VZV infection. Moreover, she had severe abdominal pain, requiring fentanyl administration. Similarly, she also received acyclovir (10 mg/kg/day).

Blood tests after admission showed that both VZV-immunoglobulin (Ig)M and VZV-IgG were positive;similarly, blood VZV DNA was elevated. There was an increase in antinuclear antibodies; however, other specific antibody tests were negative (Table 2). Her abdominal pain gradually improved, and on day 5 , she no longer required fentanyl. Her vital signs or blood tests did not deteriorate, and her abdominal pain was alleviated by

Table 1 Laboratory data at the emergency department

\begin{tabular}{|c|c|c|c|c|c|}
\hline Biochemistry & Value & Normal range & CBC / coagulopathy & Value & Normal range \\
\hline $\mathrm{TP}, \mathrm{g} / \mathrm{dL}$ & 8 & $6.5-5.8$ & $\mathrm{WBC}, \times 10^{3} / \mu \mathrm{L}$ & 7.8 & $3.9-9.8$ \\
\hline Alb, g/dL & 4.2 & $3.9-4.9$ & $\mathrm{Neu}, \%$ & 74.5 & $30-70$ \\
\hline $\mathrm{TB}, \mathrm{mg} / \mathrm{dL}$ & 0.6 & $0.2-1.2$ & Lymph, \% & 18.5 & $19-61$ \\
\hline AST, IU/L & 24 & $8-40$ & Mono, \% & 6.5 & $2-12$ \\
\hline ALT, IU/L & 30 & $8-40$ & Eos, $\%$ & 0.5 & $0-8$ \\
\hline$A L P, I U / L$ & 182 & $100-340$ & Baso, \% & 0 & $0-2$ \\
\hline $\mathrm{LDH}, \mathrm{IU} / \mathrm{L}$ & 226 & $124-222$ & $\mathrm{RBC}, \times 10^{4} / \mu \mathrm{L}$ & 484 & $350-510$ \\
\hline Amy, IU/L & 48 & $40-135$ & $\mathrm{Hb}, \mathrm{g} / \mathrm{dL}$ & 14.8 & $11.1-15.1$ \\
\hline $\mathrm{BUN}, \mathrm{mg} / \mathrm{dL}$ & 17.1 & $8-20$ & $\mathrm{Hct}, \%$ & 41.9 & $33.5-45.1$ \\
\hline $\mathrm{Cr}, \mathrm{mg} / \mathrm{dL}$ & 0.62 & $0.4-0.8$ & $\mathrm{Plt}, \times 10^{4} / \mu \mathrm{L}$ & 16.6 & $13-37$ \\
\hline$C K, I U / L$ & 42 & $50-170$ & PT, \% & 103,4 & $80-125$ \\
\hline $\mathrm{Na}, \mathrm{mEq} / \mathrm{L}$ & 137 & $136-148$ & PT-INR & 0.98 & \\
\hline $\mathrm{K}, \mathrm{mEq} / \mathrm{L}$ & 3.3 & $3.5-5.3$ & APTT, $\%$ & 100.6 & $60-110$ \\
\hline $\mathrm{Ca}, \mathrm{mg} / \mathrm{dl}$ & 9.5 & $8-10$ & & & \\
\hline Glu, mg/dl & 138 & $70-110$ & & & \\
\hline $\mathrm{CRP}, \mathrm{mg} / \mathrm{dl}$ & 2.13 & $0-0.5$ & & & \\
\hline $\mathrm{HbA} 1 \mathrm{c}, \%$ & 6.2 & & & & \\
\hline
\end{tabular}

CBC complete blood count, VZV varicella zoster virus, TP total protein, Alb albumin, TB total bilirubin, $A S T$ aspartic aminotransferase, $A L T$ alanine aminotransferase, $A L P$ alkalin phosphatase, $L D H$ lactate dehydrogenase, $A m y$ amylase, $B U N$, blood urea nitrogen, $C r$ creatinine, $C K$ creatine kinase, Na sodium, $K$ potassium, $C a$ calcium, $G l u$ glucose, CRP C-reactive protein, HbA1c hemoglobin A1C, WBC white blood cell, Neu neutrophil, Lymph lymphocyte, Mono monocyte, Eo eosinophil, Baso basophil, $R B C$ red blood cell, $H b$ hemoglobin, $H c t$ hamatocrit, Plt platelet, $P T$ :prothorombin time, PT-INR prothorombin time-international normalized ratio, APTT activated partial thromboplastin time 


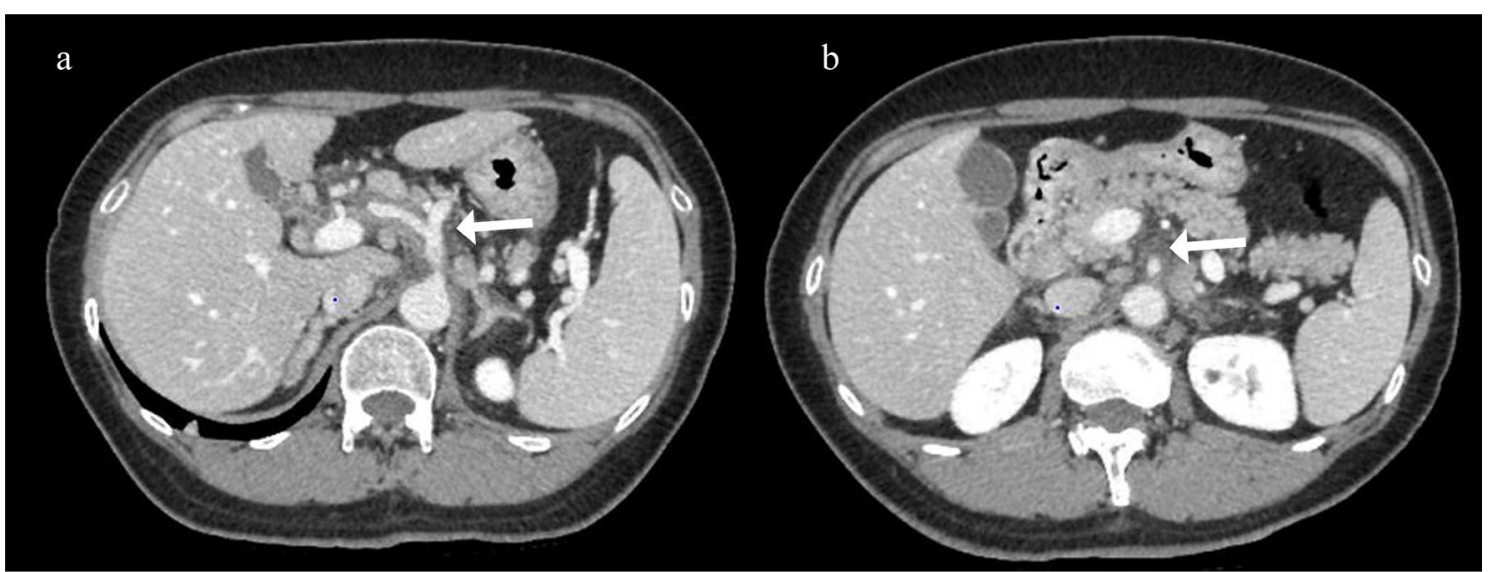

Fig. 1 Abdominal computed tomography (CT) scan at the time of emergency department visit; a Increased CT value (fat stranding) around the celiac artery and its branches. b Fat stranding around the superior mesenteric artery

Table 2 VZV and immunological test

\begin{tabular}{lll}
\hline VZV / immunological test & Value & Normal range \\
\hline VZV-IgM/EIA & 2.33 & $0-0.79$ \\
VZV-IgG/EIA & $\geq 128$ & $0-1.9$ \\
blood VZV-DNA load, Copy/mL & 710 & $0-99$ \\
$\mathrm{lgG}, \mathrm{mg} / \mathrm{dL}$ & 1646 & $870-1700$ \\
$\mathrm{lgA}, \mathrm{mg} / \mathrm{dL}$ & 427 & $110-410$ \\
$\mathrm{lgM}, \mathrm{mg} / \mathrm{dL}$ & 91 & $35-220$ \\
$\mathrm{C} 3, \mathrm{mg} / \mathrm{dL}$ & 138 & $65-135$ \\
$\mathrm{C} 4, \mathrm{mg} / \mathrm{dL}$ & 34 & $13-35$ \\
Antinuclear Ab & 80 & $0-39$ \\
Anti-RNP-Ab & $(-)$ & \\
Anti-Sm-Ab & $(-)$ & \\
Anti-SSA-Ab & $(-)$ & \\
Anti-Scl 70-Ab & $(-)$ & \\
Anti-dsDNA-Ab & $(-)$ & \\
MPO-ANCA & $(-)$ & \\
PR3-ANCA & $(-)$ &
\end{tabular}

VZV varicella zoster virus, enzyme immunoassay, IgM immunoglobulin $M$, IgG immunoglobulin $\mathrm{G}, \lg A$ immunoglobulin A, EIA enzyme immunoassay, $A b$ antibody, $R N P$ ribonucleoprotein, Sm Smith, SSA Sjogren syndrome A, Scl scleroderma, $d s D N A$ double-stranded DNA, MPO-ANCA myeloperoxidaseanti-neutrophil cytoplasmic antibody, PR3-ANCA proteinase-3-anti-neutrophil cytoplasmic antibody

oral acetaminophen. The patient was discharged on day 14.

\section{Discussion and conclusions}

This case highlighted two important clinical learning points. First, visceral disseminated VZV infection affected patients with well-controlled DM. Second, CT findings of periarterial fat stranding around the $\mathrm{CA}$ and
SMA were associated with abdominal pain in visceral disseminated VZV infection.

Visceral disseminated VZV infection has commonly been reported among immunocompromised patients, such as bone marrow or renal transplant recipients, and patients under immunosuppressive therapy [1, 8-10]. In this case, the patient had a medical history of ovarian cancer, however, the surgery was performed more than 10 years ago, and no chemotherapy was administered. Therefore, the association with ovarian cancer was considered unlikely.

Additionally, disseminated VZV infection has been reported among patients with poorly controlled DM (glycated hemoglobin $>10 \%)[6,11]$. Previous studies have identified both type 1 and type $2 \mathrm{DM}$ as risk factors for herpes zoster infection, and patients with DM had a significantly lower cell-mediated immunity to $\operatorname{VZV~}[5,12$, 13]. In this case, the patient had a glycated hemoglobin of $6.2 \%$, which indicated good control of blood glucose levels in the last one to two months. Thus, patients with DM can develop disseminated VZV infection even during periods when the blood glucose level is well-controlled. However, visceral disseminated VZV infections have not previously been reported among patients with well-controlled DM without other immunocompromising conditions, such as in this case.

Visceral disseminated VZV infection causes severe abdominal pain in $80-100 \%$ of cases [7]. VZV infection involves afferent sympathetic fibers to abdominal organs that originate from the posterior nerve roots, including cutaneous sensory fibers [14]. Disseminated VZV infection may cause inflammation of abdominal organs, such as the liver, pancreas, and intestines, due to latency and activation of the celiac ganglia, which may lead to 
frequent abdominal pain. Although, various etiologies of abdominal pain have been described, these remain unclear $[7,8]$. Despite the appearance of severe abdominal pain, abdominal CT usually shows no specific findings in most cases. Nevertheless, there have been reports of increased fat density around the CA and SMA, as in our case $[8-10,15]$. Although several conditions present with findings of periarterial fat stranding, it is unusual for fat stranding around the CA and SMA to be the isolated finding [15]. Since VZV infections have been associated with vascular disorders, especially in the cerebrovascular system [16], VZV infections may also affect visceral arteries and cause fat stranding around the CA and SMA. Furthermore, the association with visceral arteries may be a factor causing abdominal pain. Based on previous reports $[8-10,15]$, the isolated finding of fat stranding around the CA and SMA should be considered a probable cause of disseminated VZV infection.

In $20-60 \%$ of visceral disseminated VZV infections, abdominal pain appeared before the skin rash $[3,9]$. It is difficult to diagnose this disease based on a general abdominal examination. As in this case, visceral disseminated VZV infection should be considered a cause of acute abdomen in patients with a history of DM or fat stranding around the CA or SMA on abdominal CT.

In conclusion, visceral disseminated VZV infections may affect patients with well-controlled DM. CT findings of fat stranding around the CA and SMA suggest visceral disseminated VZV infection as the cause of abdominal pain. Nonetheless, further cases are needed to confirm these findings.

\section{Abbreviations}

CT: Computed tomography; CA: Celiac artery; DM: Diabetes mellitus; ED: Emergency Department; Ig: Immunoglobulin; SMA: Superior mesenteric artery; VZV: Varicella zoster virus.

\section{Acknowledgements \\ We would like to thank Editage (www.editage.com) for English language editing.}

\section{Authors' contributions}

DM and HN managed the patient and comprehensively analyzed and interpreted the patient data. DM drafted the manuscript. HN, YM and KA supervised the manuscript. All authors read and approved the final manuscript.

\section{Funding}

None.

Not applicable.

\section{Declarations}

Ethics approval and consent to participate Not applicable.

\section{Consent for publication}

Written informed consent was obtained from the patient for the publication of this case report and any accompanying images.

\section{Competing interests}

The authors declare that they have no competing interests.

Received: 14 September 2021 Accepted: 18 February 2022

Published online: 03 March 2022

\section{References}

1. Balkis MM, Ghosn S, Sharara Al, Atweh SF, Kanj SS. Disseminated varicella presenting as acute abdominal pain nine days before the appearance of the rash. Int J Infect Dis. 2009;13:e93-5.

2. Yagi T, Karasuno T, Hasegawa T, Yasumi M, Kawamoto S, Murakami M, et al. Acute abdomen without cutaneous signs of varicella zoster virus infection as a late complication of allogeneic bone marrow transplantation: importance of empiric therapy with acyclovir. Bone Marrow Transplant. 2000;25:1003-5.

3. Hyland JM, Butterworth J. Severe acute visceral pain from varicella zoster virus. Anesth Analg. 2003;97:1117-8 (table of contents).

4. de Jong MD, Weel JF, van Oers MH, Boom R, Wertheim-van Dillen PM. Molecular diagnosis of visceral herpes zoster. Lancet. 2001;357:2101-2.

5. Heymann AD, Chodick G, Karpati T, Kamer L, Kremer E, Green MS, et al. Diabetes as a risk factor for herpes zoster infection: results of a population-based study in Israel. Infection. 2008;36:226-30.

6. Malkud S, Patil SM. Disseminated cutaneous herpes zoster in a patient with uncontrolled diabetes mellitus. J Clin Diagn Res. 2015;9:WD01-2.

7. David DS, Tegtmeier BR, O'Donnell MR, Paz IB, McCarty TM. Visceral varicella-zoster after bone marrow transplantation: report of a case series and review of the literature. Am J Gastroenterol. 1998;93:810-3.

8. Furuto Y, Kawamura M, Namikawa A, Takahashi H, Shibuya Y. Successful management of visceral disseminated varicella zoster virus infection during treatment of membranous nephropathy: a case report. BMC Infect Dis. 2019;19:625.

9. Yamada S, Iwasaki T, Satoh A, Tsuboi Y, Yanagi M, Takahashi T, et al. Case of visceral varicella zoster virus infection after autologous peripheral blood stem cell transplantation in which severe abdominal pain preceded the skin rash. Nihon Shokakibyo Gakkai Zasshi. 2010;107:1947-55.

10. Takada H, Kohara S, Ito T, Yoshioka H, Okada I, Kiriu N, et al. Disseminated Varicella zoster infection with abdominal pain and periarterial fat stranding in a patient taking pomalidomide. Acute Med Surg. 2020;7: e494.

11. Graue N, Grabbe S, Dissemond J. Disseminated herpes zoster in diabetes mellitus. Dtsch Med Wochenschr. 2006;131:384-6.

12. Okamoto S, Hata A, Sadaoka K, Yamanishi K, Mori Y. Comparison of varicella-zoster virus-specific immunity of patients with diabetes mellitus and healthy individuals. J Infect Dis. 2009;200:1606-10.

13. Papagianni M, Metallidis S, Tziomalos K. Herpes zoster and diabetes mellitus: a review. Diabetes Therapy. 2018;9:545-50.

14. Rau R, Fitzhugh CD, Baird K, Cortez KJ, Li L, Fisher SH, et al. Triad of severe abdominal pain, inappropriate antidiuretic hormone section, and disseminated varicella-zoster virus infection preceding cutaneous manifestations after hematopoietic stem cell transplantation: utility of PCR for early recognition and therapy. Pediatr Infect Dis J. 2008;27:265-8.

15. Spernovasilis N, Raissaki M, Papakitsou I, Pitsigavdaki S, Louka K, Tavlas E, et al. Viscral varicella-zoster virus infection presenting with severe abdomimal pain without a rash in a patient with psoriatic arthritis treated with infliximab biosimilar and steroids: a case report. Mediterr J Rheumatol. 2021;32:280-4.

16. Nagel MA, Bubak AN. Varicella zoster virus vasculopathy. J Infect Dis. 2018;218:S107-12.

\section{Publisher's Note}

Springer Nature remains neutral with regard to jurisdictional claims in published maps and institutional affiliations. 